\title{
ORIGINAL
}

ARTICLES

\section{Education and Attitudes on Medical Cannabis in Family Medicine Residency Programs}

Ann M. Philbrick, PharmD; Christine Danner, PhD; Abayomi Oyenuga, MBBS, MPH; Chrystian Pereira, PharmD; Jason Ricco, MD, MPH; Andrea Wendling, MD

\begin{abstract}
BACKGROUND AND OBJECTIVES: Medical cannabis has become increasingly prevalent in the United States, however the extent of family medicine resident education on this topic remains unknown. The objective of this study was to ascertain the current state of medical cannabis education across this population and identify patterns in education based on state legality and program director (PD) practices.
\end{abstract}

METHODS: Survey questions were part of the Council of Academic Family Medicine Educational Research Alliance (CERA) omnibus survey from May 2019 to July 2019. PDs from all Accreditation Council for Graduate Medical Education (ACGME)-accredited US family medicine residency programs received survey invitations by email.

RESULTS: A total of 251 (40.7\%) PDs responded, with 209 (83.6\% [209/250]) reporting at least 1 hour of didactic curriculum regarding cannabis. The most common context was substance misuse (mean 3.0 \pm 4.1 hours per 3 years), followed by pain management $(2.7 \pm 3.4$ hours), and management of other conditions (2.1 \pm 2.7 hours). Thirty-eight programs (15.2\% [38/250]) offered clinical experiences related to medical cannabis, and PDs who had previously prescribed or recommended medical cannabis were more likely to offer this experience $(P<.0001)$. Experiences peaked after 3 to 5 years of medical cannabis legality. PD confidence in resident counseling skills was low overall, but did increase among programs with clinical experiences $(P=.0033)$.

CONCLUSIONS: The current trajectory of medical cannabis use in the United States makes it likely that residents will care for patients interested in medical cannabis, therefore it is important residents be prepared to address this reality. Opportunities exist for improving medical cannabis education in family medicine residency programs.

(Fam Med. 2020;52(10):730-5.)

doi: 10.22454/FamMed.2020.645274

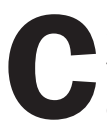
alifornia was the first state to approve the use of medical cannabis in $1996 .{ }^{1}$ While cannabis maintains its classification as a Schedule I controlled substance under federal law, the landscape of medical cannabis has dramatically changed at the state level. As of 2020, 33 states, the District of Columbia, Guam, and Puerto Rico have enacted laws allowing cannabis use for certain medical conditions. ${ }^{1}$ While prescribing cannabis is prohibited due to its Schedule I status, the majority of state medical cannabis laws involve the physician as an active participant responsible for documentation of a patient's eligibility or in the role of counseling patients regarding medical cannabis use. ${ }^{1}$

A growing body of evidence supports the efficacy of medical cannabis for specific medical conditions and associated symptoms. In 2017, the National Academy of Sciences conducted a comprehensive review and concluded that there was substantial evidence that cannabis is effective for treatment of chronic pain, as an antiemetic in the setting of chemotherapy-induced nausea and vomiting, and for patient-reported spasticity symptoms due to multiple sclerosis. ${ }^{2}$ Furthermore, there is evidence for a synergistic effect with concurrent opioid and cannabis use on chronic pain, with one study reporting a $64 \%$ reduction in the effective opioid dose for controlling pain after initiation of cannabis. ${ }^{3}$ Additional studies support the use of cannabis for posttraumatic stress disorder (PTSD), and seizure disorders. $^{4-8}$

From the Department of Pharmaceutical Care and Health Sciences, College of Pharmacy (Drs Philbrick and Pereira), and Department of Family Medicine and Community Health, Medical School, University of Minnesota, Minneapolis, MN (Drs Danner, Ricco, Philbrick, and Pereira, and Dr Oyenuga); and Department of Family Medicine, Michigan State University College of Human Medicine, East Lansing, MI (Dr Wendling). 
Along with the legal changes and growing scholarly work regarding medical use of cannabis, physician attitudes are evolving as well. ${ }^{9-11}$ In a 2017 study of Irish general practitioners (GPs), while only $28 \%$ were in favor of complete legalization of cannabis, nearly $60 \%$ supported the legalization of medical cannabis and most (between 62\%-69\%) saw cannabis as having a role in the management of pain, multiple sclerosis and palliative care. ${ }^{6}$ In a study of Australian GPs, over half supported access to medical cannabis via prescription, but only $29 \%$ felt comfortable discussing medical cannabis with their patients with most perceiving their knowledge as inadequate. ${ }^{10}$ Support for medical cannabis was stronger for use in cancer pain, palliative care and epilepsy, but much less for conditions like depression or anxiety and about a third of GPs rated medical cannabis as less harmful than other common classes of prescription medications including opioids (39\%), benzodiazepines (39\%), antidepressants $(28 \%)$, and statins $(28 \%) .{ }^{10}$ In the United States, surveys of physicians in Colorado, New York, and Washington State revealed that a majority had lingering concerns about the medical benefits and potential risks of cannabis use. Nearly all survey respondents stressed the need for medical education and formal training programs in medical cannabis. ${ }^{11-13}$

Despite this, educational resources for family physicians are clearly lacking. ${ }^{14-19}$ While one might assume that physicians in states with a longer history of available medical cannabis would have curriculum around this topic, this is currently unknown. To complicate decisions regarding educational needs, $43 \%$ of residents will practice in a state different to where they have completed their training, which may necessitate cannabis training despite local laws. ${ }^{20}$ Education regarding cannabis is especially needed now in the context of increasing support for alternative methods of pain management in response to the opioid epidemic, ${ }^{21}$ support for more comprehensive addiction therapy ${ }^{22}$ and increasing awareness of the magnitude of substance use disorders. ${ }^{5,23}$

The goal of this study was to conduct a needs assessment by ascertaining the current state of medical cannabis education across family medicine residencies in the United States. The primary objective was to understand the relationship between family medicine training in the area of medical cannabis and the history and current legal status of cannabis across states. The secondary objective was to evaluate the association between program directors' (PD) concerns about medical cannabis, their willingness to personally prescribe or recommend medical cannabis, and the type of cannabis education provided in residency. Finally, we evaluated PDs' perception of resident preparedness to counsel patients regarding the use of medical cannabis and identify perceived gaps in current curricula.

\section{Methods}

\section{Study Design}

The questions on medical cannabis were part of a larger omnibus survey conducted by the Council of Academic Family Medicine Educational Research Alliance (CERA). The methodology of the CERA Program Director Survey has previously been described in detail. ${ }^{24}$ The CERA steering committee evaluated questions for consistency with the overall subproject aim, readability, and existing evidence of reliability and validity. Pretesting was done on family medicine educators who were not part of the target population. Questions were modified following pretesting for flow, timing, and readability. The American Academy of Family Physicians Institutional Review Board approved the project in May 2019.

The sampling frame for the survey was all Accreditation Council for Graduate Medical Education (ACGME)-accredited family medicine residency PDs in the United States as identified by the Association of
Family Medicine Residency Directors. Email invitations to participate were delivered with the survey utilizing the online program SurveyMonkey. Seven follow-up emails to encourage non-respondents to participate were sent after the initial email invitation. There were 655 PDs at the time of the survey. Of these, 39 had previously opted out or blocked SurveyMonkey surveys. Therefore, the survey was emailed to 616 individuals. CERA collected data from May 2019 to July 2019.

\section{Survey Question Development}

Demographic data were obtained from the recurring questions on the CERA survey. Additionally, CERA stratified respondents using two criteria-status of cannabis legality and length of cannabis legalitybased on the survey participant's state so that researchers could remain blinded to state. Eleven questions were developed to ascertain PDs' attitudes regarding cannabis and its place in medical therapy. For the purposes of this study, medical cannabis was not specifically defined so respondents were free to interpret it as tetrahydrocannabinol (THC) predominant or cannabidiol (CBD) predominant.

\section{Data Analysis}

Demographics and other characteristics of the sample were described for all respondents. These were summarized as counts and percentages. We grouped respondents into two categories: those who had no hours dedicated to didactic curricula, and those who had approximately 1 hour or more dedicated to didactic curricula on medical cannabis. In the latter group, we summarized the number of hours dedicated to each of three didactic topics related to medical cannabis and presented as the mean \pm SD (hours).

The prevalence of clinical experiences on medical cannabis was summarized for all respondents. Due to small numbers in the "Yes, required" category, the "Yes, required" and the "Yes, elective" groups were combined 
into a single "Yes" response category for this variable. The association between the respondents' experience prescribing medical cannabis and the prevalence of clinical experiences on medical cannabis at their programs and the association between the duration of medical cannabis legalization in the respondents' state and the prevalence of clinical experiences on medical cannabis were assessed separately, using $\chi^{2}$ tests of independence.

We summarized the respondents' perception of residents' preparedness to counsel patients on medical cannabis and assessed the association between the availability of clinical experiences on medical cannabis at the respondents' residency programs and the perception of residents' preparedness to counsel using a $\chi^{2}$ test of independence. Lastly, we summarized the respondents' concerns about medical cannabis using the mean $\pm \mathrm{SD}$ of their responses to three Likert-scale questions. We performed all statistical analyses using SAS 9.4 (SAS Institute, Cary, NC) and we considered $P$ values <.05 statistically significant.

\section{Results}

The overall response rate for the survey was $42.4 \%$ (261/616) with 251 (40.7\%) PDs answering the cannabis questions. Demographic information is detailed in Table 1 . The majority of respondents were from states where cannabis is legal for medical use only (45.2\% [118/261]; Table 1). Overall survey respondents who did not choose to complete the cannabis questions differed from those that completed the cannabis questions in a few key areas. These 10 program directors were all from community-based residencies, most (80\% [8/10]) were from communities $<75,000$ in population, and almost all were directors of smaller residency programs $(<19$ residents).

A total of 209 (83.6\% [209/250]) PDs stated that they had any formal didactic curriculum regarding cannabis. Of these, cannabis in the context of substance misuse was most common with $94.7 \%$ (198/209) respondents indicating their curriculum contained this information. Didactic content regarding pain management and management of other medical conditions followed with $86.6 \%$ (181/209) and $68.9 \%$ (144/209), respectively. Hours for each topic, when present, totaled on average $3.0 \pm 4.1$ hours for substance misuse, $2.7 \pm 3.4$ hours for pain management, and 2.1 \pm 2.7 hours for other medical conditions.

Only 38 programs (15.2\% [38/250]) had a clinical experience for residents to learn how to recommend or prescribe medical cannabis, with the majority (89.5\% [34/38]) being elective experiences. PDs who have prescribed or recommended cannabis in the past were more likely to have clinical experiences for their residents compared to those who had not prescribed or recommended cannabis (37.3\% [22/59] vs $8.42 \%$ [16/190], $P<.0001)$. Additionally, clinical educational opportunities were greatest after medical cannabis had been legal for 3 to 5 years in a state, and then began to taper off (Table 2).

PDs overwhelmingly felt that their residents would not be able to effectively counsel on the medical use of cannabis postgraduation, with $60 \%$ (150/250) of respondents answering strongly disagree or disagree on a Likert scale. This changed, however, when the presence of clinical experiences were taken into consideration. PDs from programs with clinical experiences were more likely to answer agree or strongly agree (29\% [11/38] vs $9.9 \%[21 / 212], P=.0053)$.

PDs were also surveyed about their concerns regarding the use of medical cannabis. The most common concerns were its impact on mental health and potential for misuse or abuse, followed by safety concerns (eg, drug interactions), limited evidence of therapeutic benefit, and federal regulations (Table 3).

\section{Discussion}

Overall, the results of this study showed there is a deficiency of both didactic content and clinical opportunities for family medicine residents regarding the use of medical cannabis. This is consistent with prior research showing knowledge deficits surrounding medical cannabis for practicing clinicians as well as in medical residents. ${ }^{14-19}$ This has implications for a primary care workforce that is increasingly likely to encounter patients seeking clinical guidance regarding medical cannabis. Regardless of a particular state's legality of cannabis, the overall momentum of cannabis use in the United States is trending toward increased use and overall acceptance. ${ }^{25}$ Such a trend highlights the importance for family physicians to be well versed in its potential indications and safety concerns to effectively counsel patients on possible risks and benefits.

To address these gaps, didactic education could be either locally cultivated, or an emphasis placed on providing more centralized resources in the Society of Teachers of Family Medicine (STFM) Digital Resource Library. ${ }^{26}$ Resources could include teaching modules on risks and benefits, clinical case studies, recorded patient simulations, and journal club article recommendations. Developing a centralized repository may also provide resources for program directors who have had less experience counseling on medical cannabis use, a characteristic that was associated with fewer clinical learning opportunities for residents.

PDs from programs that offered clinical experiences in medical cannabis use were more confident with resident skills in this area, however only $15 \%$ of responding programs offered such an experience. In general, clinical experiences within a program should fit the specific legal reality of the program's state. For example, in Minnesota, medical cannabis is not available in combustible forms but is available in a vaporization oil option, ${ }^{27}$ therefore a clinical experience might be tailored to review indications and relevant risks for available formulations. However, it may be helpful to include some 
Table 1: Characteristics of Family Medicine Residency Program Directors and Residency Programs in the United States (Total Survey Respondent $\mathrm{N}=261$, Unless Otherwise Noted)

\begin{tabular}{|c|c|c|}
\hline Variable & $\mathbf{n}$ & $\%$ \\
\hline \multicolumn{3}{|c|}{ Describe the type of residency program you direct: } \\
\hline Community-based, university-affiliated & 156 & 59.8 \\
\hline Community-based, nonaffiliated & 51 & 19.5 \\
\hline University-based & 42 & 16.1 \\
\hline Other & 8 & 3.07 \\
\hline Military & 4 & 1.53 \\
\hline \multicolumn{3}{|c|}{ In what state is your residency program located? } \\
\hline East North Central (WI, MI, OH, IN, or IL) & 51 & 19.5 \\
\hline Middle Atlantic (NY, PA, or NJ) & 40 & 15.3 \\
\hline South Atlantic (PR, FL, GA, SC, NC, VA, DC, WV, DE, or MD) & 37 & 14.2 \\
\hline Pacific (WA, OR, CA, AK, or HI) & 32 & 12.3 \\
\hline West North Central (ND, MN, SD, IA, NE, KS, or MO) & 27 & 10.3 \\
\hline West South Central (OK, AR, LA, or TX) & 26 & 9.96 \\
\hline Mountain (MT, ID, WY, NV, UT, AZ, CO, or NM) & 25 & 9.58 \\
\hline East South Central (KY, TN, MS, or AL) & 15 & 5.75 \\
\hline New England (NH, MA, ME, VT, RI, or CT) & 8 & 3.07 \\
\hline \multicolumn{3}{|c|}{ What is the approximate size of the community in which your program is located? } \\
\hline Less than 30,000 & 33 & 12.6 \\
\hline 30,000 to 74,999 & 56 & 21.5 \\
\hline 75,000 to 149,000 & 47 & 18.0 \\
\hline 150,000 to 499,999 & 56 & 21.5 \\
\hline 500,000 to 1 million & 37 & 14.2 \\
\hline More than 1 million & 32 & 12.3 \\
\hline \multicolumn{3}{|c|}{ How many residents (total complement) were in your program as of October $2018 ?(n$, missing=4) } \\
\hline$<19$ & 115 & 44.8 \\
\hline 19-31 & 101 & 39.3 \\
\hline$>31$ & 41 & 16.0 \\
\hline \multicolumn{3}{|c|}{ Is cannabis legal in respondent's state? } \\
\hline Cannabis legal for recreational and medical & 61 & 23.4 \\
\hline Cannabis legal for medical only & 118 & 45.2 \\
\hline Cannabis not legal for any use, but CBD products can be used & 74 & 28.4 \\
\hline Neither cannabis nor CBD is legal for any use & 8 & 3.07 \\
\hline \multicolumn{3}{|c|}{ Duration of Medical Cannabis Legalization in Respondent's State } \\
\hline 0 years & 82 & 31.4 \\
\hline $0-2$ years & 16 & 6.13 \\
\hline 3-5 years & 83 & 31.8 \\
\hline 6-10 years & 19 & 7.28 \\
\hline$>10$ years & 61 & 23.4 \\
\hline \multicolumn{3}{|c|}{ Do you have any time allocated to didactic curricula on cannabis? $(n=250)$} \\
\hline Yes ( $>0$ hours on at least one topic) & 209 & 83.6 \\
\hline No (0 hours on all three topics) & 38 & 15.2 \\
\hline Did not answer & 3 & 1.2 \\
\hline \multicolumn{3}{|c|}{ Have you ever prescribed or recommended medical cannabis? $(n=250)$} \\
\hline Yes & 60 & 24.0 \\
\hline No & 190 & 76.0 \\
\hline
\end{tabular}


Table 2: Association of the Duration of Medical Cannabis Legalization and the Availability of Clinical Educational Opportunities Within 250 US Family Medicine Residency Programs

\begin{tabular}{|c|c|c|c|}
\hline \multirow{2}{*}{$\begin{array}{l}\text { Duration of Medical Cannabis Legalization } \\
\text { in Respondent's State }\end{array}$} & \multicolumn{3}{|c|}{$\begin{array}{l}\text { Do you have any clinical experiences for residents to learn how } \\
\text { to prescribe/recommend cannabis in a medical capacity? }\end{array}$} \\
\hline & $\begin{array}{l}\text { Yes, "Required" or "Elective" } \\
\text { n, (\%) }\end{array}$ & $\begin{array}{c}\text { No } \\
n,(\%)\end{array}$ & $P$ Value * \\
\hline 0 & $3(3.85)$ & $75,(96.2)$ & $<.0001$ \\
\hline $0-2$ years & $2(12.5)$ & $14,(87.5)$ & \\
\hline $3-5$ years & $24(31.2)$ & $53,(68.8)$ & \\
\hline $6-10$ years & $4(21.1)$ & $15,(79.0)$ & \\
\hline$>10$ years & 5 (8.33) & $55,(91.7)$ & \\
\hline
\end{tabular}

$* \chi^{2}$ test of independence

Table 3: Distribution of 251 Family Medicine Residency Program Directors' Concerns Regarding Medical Cannabis Use in the United States

\begin{tabular}{|l|c|c|c|c|c|}
\hline \multicolumn{2}{|c|}{$\begin{array}{l}\text { Q33: On a scale of 1-5, how concerned are you about the } \\
\text { following factors regarding the use of medical cannabis? }\end{array}$} & \multirow{N}{*}{ N } & \multicolumn{4}{c|}{ Summary } \\
\cline { 3 - 6 } & & Mean (SD) & Median (Q1, Q3) & Min. & Max. \\
\hline Federal regulation related to cannabis & 250 & $3.2(1.3)$ & $3.0(2.0,4.0)$ & 1.0 & 5.0 \\
\hline Limited evidence of therapeutic benefits from cannabis use & 251 & $3.6(1.2)$ & $4.0(3.0,5.0)$ & 1.0 & 5.0 \\
\hline Safety concerns of cannabis use (eg, drug interactions...) & 251 & $3.7(1.2)$ & $4.0(3.0,5.0)$ & 1.0 & 5.0 \\
\hline Impact on mental health and potential for misuse or addiction & 251 & $3.9(1.1)$ & $4.0(3.0,5.0)$ & 1.0 & 5.0 \\
\hline
\end{tabular}

broader discussions within these clinical experiences, including public health and health policy implications, especially as many residents may seek employment outside of the state where their residency is located. ${ }^{20}$ For programs without clinical opportunities, case studies from a centralized repository or standardized patient simulation might be educational options.

A surprising finding was that didactic educational opportunities seemed to decline after medical cannabis had been legal for 3 to 5 years per state law. One theory is that this is the typical time frame in which a state transitions from medical use only to both medical and recreational use. The subsequent decrease in didactic opportunities could represent changing attitudes regarding usefulness with the shift from a patient needing a physician to prescribe or recommend a product to being able to obtain it without this step, but this was not directly assessed in this study. This study had limitations. The response rate of $40 \%$ may not represent the opinions of all family medicine residency PDs, and therefore may not be generalizable. Additionally, given the mismatch between state and federal laws, some PDs may have chosen not to answer these questions because of fear of legal action, which could have affected overall results. Our survey collected self-reported information, rather than measuring actual practice, and as such is subject to recall bias and misrepresentation. Although tested, questions were not validated prior to implementation. Finally, medical cannabis was not defined in this survey, so respondents were open to interpret it as tetrahydrocannabinol (THC)-predominant or cannabidiol (CBD)-predominant, which may have affected how the questions were answered.

\section{Conclusion}

There are gaps in didactic and clinical medical cannabis education in family medicine residency programs. The current trajectory of medical cannabis use in the United States indicates that cannabis is becoming more common for both medical and recreational use. It is important that the next generation of medical residents be prepared to address this reality in their future practice. 
ACKNOWLEDGMENT: The authors thank Sherri Fong, MPH, for her assistance with this study

CORRESPONDING AUTHOR: Address correspondence to Dr Ann M. Philbrick, 308 Harvard Street SE, Minneapolis, MN 55455. 612-626-8053. Fax: 612-625-9931. philb020@umn.edu.

\section{References}

1. National Conference of State Legislatures. State medical marijuana laws. http://www.ncsl. org/research/health/state-medical-marijuanalaws.aspx. Accessed: April 29, 2020.

2. National Academies of Sciences, Engineering, and Medicine. 2017. The Health Effects of Cannabis and Cannabinoids: The Current State of Evidence and Recommendations for Research. Washington, DC: The National Academies Press.

3. Boehnke KF, Litinas E, Clauw DJ. Medical cannabis use is associated with decreased opiate medication use in a retrospective crosssectional survey of patients with chronic pain. J Pain. 2016;17(6):739-744.

4. Hoch E, Niemann D, von Keller R, et al. How effective and safe is medical cannabis as a treatment of mental disorders? A systematic review. Eur Arch Psychiatry Clin Neurosci. 2019;269(1):87-105.

5. Cohen J, Wei Z, Phang J, Laprairie RB, Zhang Y. PHang J, Laprairie RB, Zhang Y. Cannabinoids and emerging therapy for posttraumatic stress disorder and substance use disorders. $J$ Clin Neurophysiol. 2020;37(1):28-34

6. Hindocha C, Cousijn J, Rall M, Bloomfield MAP. The Effectiveness of Cannabinoids in the Treatment of Posttraumatic Stress Disorder (PTSD): A Systematic Review. J Dual Diagn. 2019;1-20; Epub ahead of print.

7. Hausman-Kedem M, Menascu S, Kramer U. Efficacy of CBD-enriched medical cannabis for treatment of refractory epilepsy in children and adolescents - An observational, longitudinal study. Brain Dev. 2018;40(7):544-551.
8. Epidiolex package insert. Greenwich Biosciences, Inc. Carlsbad, CA.

9. Parmar JR, Forrest BD, Freeman RA. Medical marijuana patient counseling points for health care professionals based on trends in the medical uses, efficacy, and adverse effects of cannabis-based pharmaceutical drugs. Res Social Adm Pharm. 2016;12(4):638-654

10. Karanges EA, Suraev A, Elias N, Manocha R, McGregor IS. Knowledge and attitudes of Australian general practitioners towards medicinal cannabis: a cross-sectional survey. BMJ Open. 2018;8(7):e022101.

11. Sideris A, Khan F, Boltunova A, Cuff G Gharibo C, Doan LV. New York physicians' perspective and knowledge of the state medical marijuana program. Cannabis Cannabinoid Res. 2018;3(1):74-84.

12. Kondrad E, Reid A. Colorado family physicians' attitudes toward medical marijuana. J Am Board Fam Med. 2013;26(1):52-60.

13. Carlini BH, Garrett SB, Carter GT. Medicinal cannabis: a survey among health care providers in Washington state. Am J Hosp Palliat Care. 2017;34(1):85-91.

14. Brooks E, Gundersen DC, Flynn E, BrooksRussell A, Bull S. The clinical implications of legalizing marijuana: are physician and nonphysician providers prepared? Addict Behav 2017;72:1-7.

15. Evanoff AB, Quan T, Dufault C, Awad M, Bierut LJ. Physicians-in-training are not prepared to prescribe medical marijuana. Drug Alcohol Depend. 2017;180:151-155.

16. Ware MA, Ziemianski D. Medical education on cannabis and cannabinoids: Perspectives, challenges, and opportunities. Clin Pharmacol Ther. 2015;97(6):548-550

17. Ziemianski D, Capler R, Tekanoff R, Lacasse A, Luconi F, Ware MA. Cannabis in medicine: a national educational needs assessment among Canadian physicians. BMC Med Educ. 2015;15(1):52.
18. Ricco J, Danner C, Pereira C, Philbrick AM. "The times they are a-changin" knowledge and perceptions regarding medical cannabis in an academic family medicine department. PRiMER Peer-Rev Rep Med Educ Res. 2017;1:20.

19. Mouhamed Y, Vishnyakov A, Qorri B, et al. Therapeutic potential of medicinal marijuana: an educational primer for health care professionals. Drug Healthc Patient Saf. 2018;10:4566.

20. Fagan EB, Gibbons C, Finnegan SC, et al. Family medicine graduate proximity to their site of training: policy options for improving the distribution of primary care access. Fam Med. 2015;47(2):124-130.

21. Lucas P, Walsh Z. Medical cannabis access, use, and substitution for prescription opioids and other substances: A survey of authorized medical cannabis patients. Int J Drug Policy. 2017;42:30-35

22. Reiman A. Cannabis as a substitute for alcohol and other drugs. Harm Reduct J. 2009;6(1):35.

23. Wiese B, Wilson-Poe AR. Emerging evidence for cannabis' role in opioid use disorder. Cannabis Cannabinoid Res. 2018;3(1):179-189

24. Mainous AG III, Seehusen D, Shokar N. CAFM Educational Research Alliance (CERA) 2011 Residency Director survey: background, methods, and respondent characteristics. Fam Med. 2012;44(10):691-693.

25. Carliner H, Brown QL, Sarvet AL, Hasin DS. Cannabis use, attitudes, and legal status in the U.S.: A review. Prev Med. 2017;104:13-23.

26. Society of Teachers of Family Medicine. STFM Resource Library. https://resourcelibrary.stfm. org/home. Accessed April 29, 2020

27. Minnesota Department of Health. General information about the Minnesota Medical Cannabis Program. https://www.health.state. $\mathrm{mn}$. us/people/cannabis/about/factsheet.html Accessed April 29, 2020. 